\title{
COMPOSITION OPERATORS ON SOBOLEV SPACES AND NEUMANN EIGENVALUES
}

\author{
V. GOL'DSHTEIN, A. UKHLOV
}

\begin{abstract}
In this paper we discuss applications of the geometric theory of composition operators on Sobolev spaces to the spectral theory of non-linear elliptic operators. The lower estimates of the first non-trivial Neumann eigenvalues of the $p$-Laplace operator in cusp domains $\Omega \subset \mathbb{R}^{n}, n \geq 2$, are given.
\end{abstract}

\section{INTRODUCTION}

In the article we discuss a general method of applications of the geometric theory of composition operators on Sobolev spaces to spectral estimates of the non-linear Neumann-Laplace operators, proposed in our recent works. Suppose that in a bounded domain $\Omega \subset \mathbb{R}^{n}$ the following Sobolev-Poincaré inequality

$$
\inf _{c \in \mathbb{R}}\left\|f-c\left|L_{p}(\Omega)\left\|\leq B_{p, p}(\Omega)\right\| \nabla f\right| L_{p}(\Omega)\right\|, \quad f \in W_{p}^{1}(\Omega) .
$$

holds with the Poincaré constant $B_{p, p}(\Omega)$. Then the first nontrivial Neumann eigenvalue $\mu_{p}(\Omega)$ of the $p$-Laplace operator

$$
-\Delta_{p} u=-\operatorname{div}\left(|\nabla u|^{p-2} \nabla u\right), p>1,
$$

can be characterized as $\mu_{p}(\Omega)=B_{p, p}^{-p}(\Omega)$ (see, for example, [6]).

Exact calculations of Neumann eigenvalues are known in very limited number of domains and so estimates of $\mu_{p}(\Omega)$ represent an important part of the modern spectral theory (see, for example, [2, 6, 12, 28, 24, 36]). We suggest spectral estimates of the first nontrivial Neumann eigenvalue $\mu_{p}(\Omega)$ of the $p$-Laplace operator in terms of the (weak) quasiconformal geometry of a domain $\Omega$.

The classical upper estimate for the first nontrivial Neumann eigenvalue of the Laplace operator

$$
\mu_{2}(\Omega) \leq \mu_{2}\left(\Omega^{*}\right)=\frac{p_{n / 2}^{2}}{R_{*}^{2}}
$$

was proved by Szegö [47 for simply connected planar domains via a conformal mappings technique ("the method of conformal normalization") and by Weinberger [59] for domains in $\mathbb{R}^{n}$. In this inequality $p_{n / 2}$ denotes the first positive zero of the function $\left(t^{1-n / 2} J_{n / 2}(t)\right)^{\prime}$, and $\Omega^{*}$ is an $n$-ball of the same $n$-volume as $\Omega$ with $R_{*}$ as its radius. In particular, if $n=2$, we have $p_{1}=j_{1,1}^{\prime} \approx 1.84118$ where $j_{1,1}^{\prime}$ denotes the first positive zero of the derivative of the Bessel function $J_{1}$.

\footnotetext{
${ }^{0}$ Key words and phrases: Sobolev spaces, Neumann eigenvalues, Quasiconformal mappings ${ }^{0} 2000$ Mathematics Subject Classification: 35P15, 46E35, 30C65.
} 
Is convex domains $\Omega \subset \mathbb{R}^{n}, n \geq 2$, the classical lower estimates of the linear Neumann-Laplace operator $(\mathrm{p}=2)$ [44] states that

$$
\mu_{2}(\Omega) \geq \frac{\pi^{2}}{d(\Omega)^{2}}
$$

where $d(\Omega)$ is a diameter of a convex domain $\Omega$. Similar estimates for $p \neq 2$ were obtained much later [28]:

$$
\mu_{p}(\Omega) \geq\left(\frac{\pi_{p}}{d(\Omega)}\right)^{p}
$$

where

$$
\pi_{p}=2 \int_{0}^{(p-1)^{\frac{1}{p}}} \frac{d t}{\left(1-t^{p} /(p-1)\right)^{\frac{1}{p}}}=2 \pi \frac{(p-1)^{\frac{1}{p}}}{p \sin (\pi / p)} .
$$

Unfortunately in non-convex domains $\mu_{p}(\Omega)$ can not be estimated in the terms of Euclidean diameters. It can be seen by considering a domain consisting of two identical squares connected by a thin corridor [3]. In our previous works [21, 22] we returned to a conformal mappings techniques (that was used in [47]) in a framework of composition operators on Sobolev spaces. It permit us to obtain lower estimates of $\mu_{2}(\Omega)$ in the terms of the hyperbolic (conformal) radius of $\Omega$ for a large class of general (non necessary convex) domains $\Omega \subset \mathbb{R}^{2}$.

In the case of space domains conformal mappings does not allow to obtain spectral estimates of the Neumann-Laplace operator and we use a generalization of conformal mappings such as weak $p$-quasiconformal mappings [23]. The aim of the present work is to obtain lower estimates of the Neumann eigenvalues of the $p$-Laplace operator in the terms of general weak $(p, q)$-quasiconformal geometry of domains. We use one more time the geometric theory of composition operators on Sobolev spaces [15, 48, with applications to the (weighted) Poincaré-Sobolev inequalities [14, 19].

The paper is organized as follows: The capacitory description of Sobolev spaces and a short survey of the geometric theory of composition operators on Sobolev spaces are presented in section 2 . In section 3 we give a general method of applications of composition operators to the Sobolev-Poincaré inequalities and apply them to lower estimates of the first nontrivial Neumann eigenvalue for the $p$-Laplace operator.

\section{Composition operators on Sobolev Spaces}

2.1. Sobolev spaces. Let $E$ be a measurable subset of $\mathbb{R}^{n}, n \geq 2$. The Lebesgue space $L_{p}(E), 1 \leq p \leq \infty$, is defined as a Banach space of $p$-summable functions $f: E \rightarrow \mathbb{R}$ equipped with the following norm:

$$
\left\|f \mid L_{p}(E)\right\|=\left(\int_{E}|f(x)|^{p} d x\right)^{\frac{1}{p}}, \quad 1 \leq p<\infty,
$$

and

$$
\left\|f\left|L_{\infty}(E) \|=\operatorname{ess} \sup _{x \in E}\right| f(x) \mid, \quad p=\infty\right.
$$


If $\Omega$ is an open subset of $\mathbb{R}^{n}$, the Sobolev space $W_{p}^{1}(\Omega), 1 \leq p \leq \infty$, is defined as a Banach space of locally integrable weakly differentiable functions $f: \Omega \rightarrow \mathbb{R}$ equipped with the following norm:

$$
\left\|f\left|W_{p}^{1}(\Omega)\|=\| f\right| L_{p}(\Omega)\right\|+\left\|\nabla f \mid L_{p}(\Omega)\right\|,
$$

where $\nabla f$ is the weak gradient of the function $f$, i. e. $\nabla f=\left(\frac{\partial f}{\partial x_{1}}, \ldots, \frac{\partial f}{\partial x_{n}}\right)$,

$$
\int_{\Omega} f \frac{\partial g}{\partial x_{i}} d x=-\int_{\Omega} \frac{\partial f}{\partial x_{i}} g d x, \quad \forall g \in C_{0}^{\infty}(\Omega), \quad i=1, \ldots, n .
$$

As usual, $C_{0}^{\infty}(\Omega)$ is the space of infinitely smooth functions with a compact support.

The homogeneous seminormed Sobolev space $L_{p}^{1}(\Omega), 1 \leq p \leq \infty$, is defined as a space of locally integrable weakly differentiable functions $f: \Omega \rightarrow \mathbb{R}$ equipped with the following seminorm:

$$
\left\|f\left|L_{p}^{1}(\Omega)\|=\| \nabla f\right| L_{p}(\Omega)\right\| .
$$

Recall the notion of the $p$-capacity of a set $E \subset \Omega$. Let $\Omega$ be a domain in $\mathbb{R}^{n}$ and a compact $F \subset \Omega$. The $p$-capacity of the compact $F$ is defined by

$$
\operatorname{cap}_{p}(F ; \Omega)=\inf \left\{\| f\left|L_{p}^{1}(\Omega)\right|^{p}, f \geq 1 \text { on } F, f \in C_{0}(\Omega) \cap L_{p}^{1}(\Omega),\right.
$$

where $C_{0}(\Omega)$ is the space of continuous functions with a compact support.

By the similar way we can define $p$-capacity of open sets.

For arbitrary set $E \subset \Omega$ we define an inner $p$-capacity as

$$
\underline{\operatorname{cap}}_{p}(E ; \Omega)=\sup \left\{\operatorname{cap}_{p}(e ; \Omega), e \subset E \subset \Omega, e \text { is a compact }\right\},
$$

and an outer $p$-capacity as

$$
\overline{\operatorname{cap}}_{p}(E ; \Omega)=\inf \left\{\operatorname{cap}_{p}(U ; \Omega), E \subset U \subset \Omega, U \text { is an open set }\right\} .
$$

A set $E \subset \Omega$ is called $p$-capacity measurable, if $\underline{\operatorname{cap}}_{p}(E ; \Omega)=\overline{\operatorname{cap}}_{p}(E ; \Omega)$. The value

$$
\operatorname{cap}_{p}(E ; \Omega)=\underline{\operatorname{cap}}_{p}(E ; \Omega)=\overline{\operatorname{cap}}_{p}(E ; \Omega)
$$

is called the $p$-capacity of the set $E \subset \Omega$.

The notion of $p$-capacity permits us to refine the notion of Sobolev functions. Let a function $f \in L_{p}^{1}(\Omega)$. Then refined function

$$
\tilde{f}(x)=\lim _{r \rightarrow 0} \frac{1}{|B(x, r)|} \int_{B(x, r)} f(y) d y
$$

is defined quasieverywhere i. e. up to a set of $p$-capacity zero and it is absolutely continuous on almost all lines [41. This refined function $\tilde{f} \in L_{p}^{1}(\Omega)$ is called a unique quasicontinuous representation (a canonical representation) of function $f \in L_{p}^{1}(\Omega)$. Recall that a function $\tilde{f}$ is termed quasicontinuous if for any $\varepsilon>0$ there is an open set $U_{\varepsilon}$ such that the p-capacity of $U_{\varepsilon}$ is less than $\varepsilon$ and on the set $\Omega \backslash U_{\varepsilon}$ the function $\tilde{f}$ is continuous (see, for example [26, 41]). In what follows we will use the quasicontinuous (refined) functions only.

Note that the first weak derivatives of the function $f$ coincide almost everywhere with the usual partial derivatives (see, e.g., 41] ). 
2.2. Composition operators. Let $\Omega$ and $\widetilde{\Omega}$ be domains in $\mathbb{R}^{n}, n \geq 2$. We say that a homeomorphism $\varphi: \Omega \rightarrow \widetilde{\Omega}$ induces a bounded composition operator

$$
\varphi^{*}: L_{p}^{1}(\widetilde{\Omega}) \rightarrow L_{q}^{1}(\Omega), \quad 1 \leq q \leq p \leq \infty,
$$

by the composition rule $\varphi^{*}(f)=f \circ \varphi$, if for any function $f \in L_{p}^{1}(\widetilde{\Omega})$, the composition $\varphi^{*}(f) \in L_{q}^{1}(\Omega)$ is defined quasi-everywhere in $\Omega$ and there exists a constant $K_{p, q}(\Omega)<\infty$ such that

$$
\left\|\varphi^{*}(f)\left|L_{q}^{1}(\Omega)\left\|\leq K_{p, q}(\Omega)\right\| f\right| L_{p}^{1}(\widetilde{\Omega})\right\| .
$$

Composition operators on Sobolev spaces arise in [40] as operators generated by the class of sub-areal mappings. In [54] in the frameworks of Reshetnyak's problem (1968) was proved that a homeomorphism $\varphi: \Omega \rightarrow \widetilde{\Omega}$ generates an isomorphism of Sobolev spaces $L_{n}^{1}(\Omega)$ and $L_{n}^{1}(\widetilde{\Omega})$ if and only if $\varphi$ is a quasiconformal mapping. These works motivated the study of geometric properties of mappings which generate isomorphisms (bounded operators) of Sobolev type spaces. In [55] was proved that a homeomorphism $\varphi: \Omega \rightarrow \widetilde{\Omega}$ generates an isomorphism of Sobolev spaces $L_{p}^{1}(\Omega)$ and $L_{p}^{1}(\widetilde{\Omega}), p>n$, if and only if $\varphi$ is a bi-Lipschitz mapping. This result was extended to the cases $n-1<p<n$ in [18] and the case $1 \leq p<n$ in [37.

Remark 2.1. Recall that a homeomorphism $\varphi$ is called a bi-Lipschitz homeomorphism if $\varphi$ and $\varphi^{-1}$ are locally Lipschitz mappings with uniformly bounded local Lipschitz constants.

Mappings generates isomorphisms of Besov spaces was considered in [51], NikolskiiBesov spaces and Lizorkin-Triebel spaces were considered in 52. In [42] the theory of multipliers was applied to the change of variable problem in Sobolev spaces.

The composition operators are not necessary isomorphisms even if they induced by diffeomorphisms or homeomorphisms of Euclidean domains. It means that the composition problem can be reformulated by a more flexible way: describe classes of homeomorphisms that induce bounded composition operators on Sobolev spaces. In the case of Sobolev spaces with the first weak derivatives it can be formulated as a characterization of homeomorphism $\varphi: \Omega \rightarrow \widetilde{\Omega}$ that generate composition operators

$$
\varphi^{*}: L_{p}^{1}(\widetilde{\Omega}) \rightarrow L_{q}^{1}(\Omega), \quad 1 \leq q \leq p \leq \infty,
$$

generated by the standard composition rule $\varphi^{*}(f)=f \circ \varphi$.

Analytical characteristics of mappings generate bounded compositions operators on Sobolev spaces are given in terms of weak derivatives of the mappings. Let a mapping $\varphi: \Omega \rightarrow \mathbb{R}^{n}$ be weakly differentiable in $\Omega$. Then the formal Jacobi matrix $D \varphi(x)$ and its determinant (Jacobian) $J(x, \varphi)$ are well defined at almost all points $x \in \Omega$. The norm $|D \varphi(x)|$ of the matrix $D \varphi(x)$ is the norm of the corresponding linear operator. We will use the same notation for this matrix and the corresponding linear operator.

Recall that a mapping $\varphi: \Omega \rightarrow \mathbb{R}^{n}$ possesses the Luzin $N$-property if an image of any set of measure zero has measure zero. Lipschitz mapping possess the Luzin $N$-property.

In the case $p=q \neq n$ an analytic description was obtained in [50] using a notion of mappings of finite distortion introduced in [56]: a weakly differentiable mapping is called a mapping of finite distortion if $|D \varphi(x)|=0$ a. e. on the set 
$Z=\{x \in \Omega: J(x, \varphi)=0\}$. This property becomes useful especially for the case $p \neq q$.

In [15] it was obtained the geometric description of composition operators for $n-1<p=q<\infty$ without using the finite distortion property. In the case $1<p<$ $n-1$ there are proofs [15] with additional apriori properties of homeomorphisms.

Let us reformulate the main result of [15].

Theorem 2.2. [15] A homeomorphism $\varphi: \Omega \rightarrow \widetilde{\Omega}$ between two domains $\Omega$ and $\widetilde{\Omega}$ induces a bounded composition operator

$$
\varphi^{*}: L_{p}^{1}(\widetilde{\Omega}) \rightarrow L_{p}^{1}(\Omega), \quad n-1<p<\infty,
$$

if and only if

$$
M_{p}^{(\lambda)}(\varphi ; \Omega)=\sup _{x \in \Omega} \limsup _{r \rightarrow 0} \frac{L_{\varphi}^{p}(x, r) r^{n-p}}{|\varphi(B(x, \lambda r))|}<\infty
$$

for some $\lambda>1$ if $n-1<p<n$ and $\lambda=1$ if $n \leq p<\infty$, where $L_{\varphi}(x, r)=$ $\max _{|x-y|=r}|\varphi(x)-\varphi(y)|$.

On the base of this result the notion of $p$-quasiconformal mappings was introduced for $n-1<p<\infty$. In the case $1<p<n-1$ this result is correct under an additional apriori property of weak differentiability of $\varphi$ [15].

Recall that a weakly differentiable homeomorphism $\varphi: \Omega \rightarrow \widetilde{\Omega}$ is called a weak $p$-quasiconformal mapping if there exists a constant $K_{p}<\infty$ such that

$$
|D \varphi(x)|^{p} \leq K_{p}^{p} \mid J(x, \varphi \mid \text { for almost all } x \in \Omega .
$$

The class of weak $p$-quasiconformal mappings are natural generalization of quasiconformal mappings and for $p=n$ these classes coincide.

Note that planar conformal mapping preserve the Dirichlet energy integral

$$
\int_{\Omega}|\nabla f(x)|^{2} d x
$$

because $|D \varphi(x)|^{2}=\mid J(x, \varphi \mid$ in $\Omega$.

In the space $\mathbb{R}^{3}$ conformal mappings don't connected to the Dirichlet energy integral, but the class of weak 2-conformal mappings (co-conformal mappings [23]) generates bounded composition operators on the Dirichlet spaces. It is a reason for detailed study of weak 2-quasiconformal mappings that looks as an appropriate class for possible applications to elliptic equations as was mentioned in [23].

The case $p \neq q$ is more complicated and in this case the composition operators theory is based on the countable-additive property of the norm of composition operators defined on open subsets of $\Omega$ [48] (see also [58]). The main result of [48] gives an analytic description of composition operators on Sobolev spaces (see, also [58]) and asserts that

Theorem 2.3. 48 A homeomorphism $\varphi: \Omega \rightarrow \widetilde{\Omega}$ between two domains $\Omega$ and $\widetilde{\Omega}$ induces a bounded composition operator

$$
\varphi^{*}: L_{p}^{1}(\widetilde{\Omega}) \rightarrow L_{q}^{1}(\Omega), \quad 1 \leq q<p<\infty,
$$


if and only if $\varphi \in W_{1, \mathrm{loc}}^{1}(\Omega)$, has finite distortion, and

$$
K_{p, q}(\varphi ; \Omega)=\left(\int_{\Omega}\left(\frac{|D \varphi(x)|^{p}}{|J(x, \varphi)|}\right)^{\frac{q}{p-q}} d x\right)^{\frac{p-q}{p q}}<\infty .
$$

Let us remark one more time that in the framework of two previous theorems function of spaces $L_{p}^{1}$ are quasicontinuous. If the concept of quasicontinuity is not used it can lead to wrong conclusions. In [29] by an analogy with Lebesgue spaces, Sobolev functions were defined up to set s measure zero (not up to sets of $p$-capacity zero). Recall that by the Sobolev embedding theorem (see, for example, [26, 41]) any function $f \in W_{p}^{1}(\widetilde{\Omega}), p>n$, has an unique continuous representation [41] and it is natural to consider only these continuous functions (in this case points have nonzero $p$-capacity, $p>n$ ).

Because weak $p$-quasiconformal mappings and weak $(p, q)$-quasiconformal mappings not necessary have the Luzin $N^{-1}$-property, i.e. pre-images of sets of a measure zero not necessary have a measure zero, this observation was used in [29] for the wrong conclusion that Theorem 2.2 and Theorem 2.3 are not correct (especially in its sufficiency parts). We put here this detailed explanation because the mistake in 29] was not fixed up to this moment.

By an analogy with a notion of weak $p$-quasiconformal case homeomorphisms which satisfy conditions of Theorem 2.3 are called (weak) $(p, q)$-quasiconformal mappings [57] or mappings of bounded $(p, q)$-distortion [49]. The geometric description similar (2.2) of weak $(p, q)$-quasiconformal mappings in the case $q>n-1$ was obtained in [57.

Composition operators on Sobolev spaces have applications to spectral problems of elliptic equations. These applications are based on the Sobolev type embedding theorems [14, 19]. The following diagram illustrate the main idea of these applications:

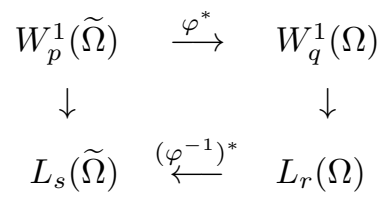

Here the operator $\varphi^{*}$ defined by the composition rule $\varphi^{*}(f)=f \circ \varphi$ is a bounded composition operator on Sobolev spaces induced by a homeomorphism $\varphi$ of a "good" domain $\Omega$ (for example the unit ball) to a "bad" domain $\widetilde{\Omega}$ and the operator $\left(\varphi^{-1}\right)^{*}$ defined by the composition rule $\left(\varphi^{-1}\right)^{*}(f)=f \circ \varphi^{-1}$ is a bounded composition operator on Lebesgue spaces induced by the inverse homeomorphism.

This approach allows us to obtain estimates of stability of constants in SobolevPoincare inequalities in domains, that in its turn give us opportunity to estimates stability of Neumann eigenvalues of the $p$-Laplace operators.

In our recent works [8, 9, 16, 21, 22] the spectral stability problem and the lower estimates of Neumann eigenvalues in planar domains were studied. In space domains our results are more modest. Some spectral estimates in space non convex domains were obtained [23] with the help of weak $p$-quasiconformal mappings theory. 
The geometric theory of composition operators on Sobolev spaces is closely connected with the $A_{p, q}$-Ball's classes [4, 5] and has applications in non-linear elasticity (see, for example, [11, 20, 53).

In the last decade the composition operators theory on generalizations of Sobolev spaces, like Besov spaces and Triebel-Lizorkin spaces, [27, 32, 33, 34, 43, was under consideration. These types of composition operators have applications to the Calderón inverse conductivity problem [10].

2.3. Composition operators and capacity inequalities. Composition operators on Sobolev spaces allow an alternative capacitory description. Recall the notion of a variational $p$-capacity [17].

A condenser in the domain $\Omega \subset \mathbb{R}^{n}$ is the pair $\left(F_{0}, F_{1}\right)$ of connected closed relatively to $\Omega$ sets $F_{0}, F_{1} \subset \Omega$. A continuous function $f \in L_{p}^{1}(\Omega)$ is called an admissible function for the condenser $\left(F_{0}, F_{1}\right)$, if the set $F_{i} \cap \Omega$ is contained in some connected component of the set $\operatorname{Int}\{x \mid u(x)=i\}, i=0,1$. We call as the $p$-capacity of the condenser $\left(F_{0}, F_{1}\right)$ relatively to domain $\Omega$ the following quantity:

$$
\operatorname{cap}_{p}\left(F_{0}, F_{1} ; \Omega\right)=\inf \left\|f \mid L_{p}^{1}(\Omega)\right\|^{p} .
$$

Here the greatest lower bond is taken over all functions admissible for the condenser $\left(F_{0}, F_{1}\right) \subset \Omega$. If the condenser has no admissible functions we put the capacity equal to infinity.

The following theorems give the capacitory description of the composition operators on Sobolev spaces.

Theorem 2.4. [15] Let $1<p<\infty$. A homeomorphism $\varphi: \Omega \rightarrow \widetilde{\Omega}$ generates a bounded composition operator

$$
\varphi^{*}: L_{p}^{1}(\widetilde{\Omega}) \rightarrow L_{p}^{1}(\Omega)
$$

if and only if for every condenser $\left(F_{0}, F_{1}\right) \subset \widetilde{\Omega}$ the inequality

$$
\operatorname{cap}_{p}^{1 / p}\left(\varphi^{-1}\left(F_{0}\right), \varphi^{-1}\left(F_{1}\right) ; \Omega\right) \leq K \operatorname{cap}_{p}^{1 / p}\left(F_{0}, F_{1} ; \widetilde{\Omega}\right)
$$

holds.

Theorem 2.5. 57 Let $1<q<p<\infty$. A homeomorphism $\varphi: \Omega \rightarrow \widetilde{\Omega}$ generates a bounded composition operator

$$
\varphi^{*}: L_{p}^{1}(\widetilde{\Omega}) \rightarrow L_{q}^{1}(\Omega)
$$

if and only if there exists a bounded monotone countable-additive set function $\Phi$ defined on open subsets of $\widetilde{\Omega}$ such that for every condenser $\left(F_{0}, F_{1}\right) \subset \widetilde{\Omega}$ the inequality

$$
\operatorname{cap}_{q}^{1 / q}\left(\varphi^{-1}\left(F_{0}\right), \varphi^{-1}\left(F_{1}\right) ; \Omega\right) \leq \Phi\left(\widetilde{\Omega} \backslash\left(F_{0} \cup F_{1}\right)\right)^{\frac{p-q}{p q}} \operatorname{cap}_{p}^{1 / p}\left(F_{0}, F_{1} ; \widetilde{\Omega}\right)
$$

holds.

This capacity inequalities demonstrate a close connection of mappings that generate bounded composition operators on Sobolev spaces and so-called $Q$-homeomorphisms 38. Descriptions of $Q$-homeomorphisms are based on the capacitory (moduli) distortion property are these classes were intensively studied at last decades (see, for example, [35, 45, 46]). 


\section{Spectral estimates of the $p$-Laplace operator}

In this section we give spectral estimates of the $p$-Laplace operator on the base Sobolev-Poincaré inequalities.

3.1. The general case. Recall that a bounded domain $\Omega \subset \mathbb{R}^{n}$ is called an $(r, q)$ Sobolev-Poincaré domain, $1 \leq r, q \leq \infty$, if for any function $f \in L_{q}^{1}(\Omega)$, the $(r, q)$ Sobolev-Poincaré inequality

$$
\inf _{c \in \mathbb{R}}\left\|f-c\left|L_{r}(\Omega)\left\|\leq B_{r, q}(\Omega)\right\| \nabla f\right| L_{p}(\Omega)\right\|
$$

holds.

Weak $(p, q)$-quasiconformal mappings permits us to "transfer" this property from one domain to another.

Theorem 3.1. Let a bounded domain $\Omega \subset \mathbb{R}^{n}$ be a $(r, q)$-Sobolev-Poncaré domain, $1<q \leq r<\infty$, and there exists a weak $(p, q)$-quasiconformal mapping $\varphi: \Omega \rightarrow \widetilde{\Omega}$ of a domain $\Omega$ onto a bounded domain $\widetilde{\Omega}$, possesses the Luzin $N$-property and such that

$$
M_{r}(\Omega)=\operatorname{ess} \sup _{x \in \Omega}|J(x, \varphi)|^{\frac{1}{r}}<\infty .
$$

Then in the domain $\widetilde{\Omega}$ the $(r, p)$-Sobolev-Poincaré inequality

$$
\inf _{c \in \mathbb{R}}\left(\int_{\widetilde{\Omega}}|f(x)-c|^{r} d x\right)^{\frac{1}{r}} \leq B_{r, p}(\widetilde{\Omega})\left(\int_{\widetilde{\Omega}}|\nabla f(x)|^{p} d x\right)^{\frac{1}{p}}, f \in W_{p}^{1}(\widetilde{\Omega}),
$$

holds and

$$
B_{r, p}(\widetilde{\Omega}) \leq K_{p, q}(\Omega) M_{r}(\Omega) B_{r, q}(\Omega) .
$$

Here $B_{r, q}(\Omega)$ is the best constant in the $(r, q)$-Sobolev-Poincare inequality in the domain $\Omega$.

Proof. Let $f \in L_{p}^{1}(\widetilde{\Omega})$. By the conditions of the theorem there exists a weak $(p, q)$ quasiconformal homeomorphism $\varphi: \Omega \rightarrow \widetilde{\Omega}$. Hence, the composition operator

$$
\varphi^{*}: L_{p}^{1}(\widetilde{\Omega}) \rightarrow L_{q}^{1}(\Omega)
$$

is bounded. Because $\Omega$ is a bounded $(r, q)$-Sobolev-Poincaré domain $g=\varphi^{*}(f) \in$ $W_{q}^{1}(\Omega)$.

Using the change of variable formula we obtain:

$$
\begin{aligned}
& \inf _{c \in \mathbb{R}}\left(\int_{\widetilde{\Omega}}|f(y)-c|^{r} d y\right)^{\frac{1}{r}}=\inf _{c \in \mathbb{R}}\left(\int_{\Omega}|f(\varphi(x))-c|^{r}|J(x, \varphi)| d x\right)^{\frac{1}{r}} \\
\leq & \operatorname{ess} \sup _{x \in \Omega}|J(x, \varphi)|^{\frac{1}{r}} \inf _{c \in \mathbb{R}}\left(\int_{\Omega}|f(\varphi(x))-c|^{r} d x\right)^{\frac{1}{r}}=M_{r}(\Omega) \inf _{c \in \mathbb{R}}\left(\int_{\Omega}|g(x)-c|^{r} d x\right)^{\frac{1}{r}} .
\end{aligned}
$$

Because the domain $\Omega$ is a $(r, q)$-Sobolev-Poincaré domain we have

$$
\inf _{c \in \mathbb{R}}\left(\int_{\Omega}|g(x)-c|^{r} d x\right)^{\frac{1}{r}} \leq B_{r, q}(\Omega)\left(\int_{\Omega}|\nabla g(x)|^{q} d x\right)^{\frac{1}{q}} .
$$


Hence

$$
\inf _{c \in \mathbb{R}}\left(\int_{\widetilde{\Omega}}|f(y)-c|^{r} d y\right)^{\frac{1}{r}} \leq M_{r}(\Omega) B_{r, q}(\Omega)\left\|g \mid L_{q}^{1}(\Omega)\right\| .
$$

By Theorem 2.3

$$
\left\|g\left|L_{q}^{1}(\Omega)\left\|\leq K_{p, q}(\Omega)\right\| f\right| L_{p}^{1}(\widetilde{\Omega})\right\| .
$$

Therefore

$$
\inf _{c \in \mathbb{R}}\left(\int_{\widetilde{\Omega}}|f(y)-c|^{r} d y\right)^{\frac{1}{r}} \leq K_{p, q}(\Omega) M_{r}(\Omega) B_{r, q}(\Omega)\left(\int_{\widetilde{\Omega}}|\nabla f(x)|^{p} d x\right)^{\frac{1}{p}} .
$$

The Theorem 3.1 immediately implies the following lower estimate for $\mu_{p}(\tilde{\Omega})$ :

Theorem 3.2. Suppose that there exists a $(p, q)$-quasiconformal homeomorphism $\varphi: \Omega \rightarrow \widetilde{\Omega}$, of a $(r, q)$-Sobolev-Poncaré domain $\Omega \subset \mathbb{R}^{n}$ onto $\widetilde{\Omega}$, possesses the Luzin $N$-property and such that

$$
M_{p}(\Omega)=\operatorname{ess} \sup _{x \in \Omega}|J(x, \varphi)|^{\frac{1}{p}}<\infty .
$$

Then

$$
\mu_{p}(\widetilde{\Omega}) \geq\left(K_{p, q}^{p}(\Omega) M_{p}^{p}(\Omega) B_{p, q}^{p}(\Omega)\right)^{-1} .
$$

Boundedness of a Jacobian of a weak $(p, q)$-quasiconformal mapping is a sufficient but restrictive assumption. In this case $\varphi$ is a Lipschitz mapping this condition holds but an image of a Lipschitz domain can not be a domain with external singularities.

We shall use a more flexible class of weak $(p, q)$-quasiconformal mappings with an integrable Jacobian, which allows us to map Lipschitz domains onto cusp domains.

Theorem 3.3. Let a bounded domain $\Omega \subset \mathbb{R}^{n}$ be a $(r, q)$-Sobolev-Poncaré domain, $1<q \leq r<\infty$, and there exists a weak $(p, q)$-quasiconformal homeomorphism $\varphi: \Omega \rightarrow \widetilde{\Omega}$ of a domain $\Omega$ onto a bounded domain $\widetilde{\Omega}$, possesses the Luzin $N$ property and such that

$$
M_{r, s}(\Omega)=\left(\int_{\Omega}|J(x, \varphi)|^{\frac{r}{r-s}} d x\right)^{\frac{r-s}{r s}}<\infty
$$

for some $1 \leq s<r$. Then in the domain $\widetilde{\Omega}$ the $(s, p)$-Sobolev-Poincaré inequality

$$
\left(\int_{\widetilde{\Omega}}\left|f(x)-f_{\widetilde{\Omega}}\right|^{s} d x\right)^{\frac{1}{s}} \leq B_{s, p}(\widetilde{\Omega})\left(\int_{\widetilde{\Omega}}|\nabla f(x)|^{p} d x\right)^{\frac{1}{p}}, f \in W_{p}^{1}(\widetilde{\Omega}),
$$

holds and

$$
B_{s, p}(\widetilde{\Omega}) \leq K_{p, q}(\Omega) M_{r, s}(\Omega) B_{r, q}(\Omega) .
$$

Here $B_{r, q}(\Omega)$ is the best constant in the $(r, q)$-Sobolev-Poincare inequality in the domain $\Omega$.

Proof. Let $f \in L_{p}^{1}(\widetilde{\Omega})$. By the conditions of the theorem there exists a $(p, q)$ quasiconformal homeomorphism $\varphi: \Omega \rightarrow \widetilde{\Omega}$. By Theorem 2.3 the composition operator

$$
\varphi^{*}: L_{p}^{1}(\widetilde{\Omega}) \rightarrow L_{q}^{1}(\Omega)
$$


is bounded. Because the bounded domain $\Omega$ is a $(r, q)$-Sobolev-Poncaré domain $g=\varphi^{*}(f) \in W_{q}^{1}(\Omega)$.

Using the change of variable formula and the Hölder inequality we obtain:

$$
\begin{gathered}
\inf _{c \in \mathbb{R}}\left(\int_{\widetilde{\Omega}}|f(y)-c|^{s} d y\right)^{\frac{1}{s}}=\inf _{c \in \mathbb{R}}\left(\int_{\Omega}|f(\varphi(x))-c|^{s}|J(x, \varphi)| d x\right)^{\frac{1}{s}} \\
\leq\left(\int_{\Omega}|J(x, \varphi)|^{\frac{r}{r-s}} d x\right)^{\frac{r-s}{r s}} \inf _{c \in \mathbb{R}}\left(\int_{\Omega}|f(\varphi(x))-c|^{r} d x\right)^{\frac{1}{r}} \\
=M_{r, s}(\Omega) \inf _{c \in \mathbb{R}}\left(\int_{\Omega}|g(x)-c|^{r} d x\right)^{\frac{1}{r}} .
\end{gathered}
$$

Because the domain $\Omega$ is a $(r, q)$-Sobolev-Poincaré domain the following inequality holds:

$$
\inf _{c \in \mathbb{R}}\left(\int_{\Omega}|g(x)-c|^{r} d x\right)^{\frac{1}{r}} \leq B_{r, q}(\Omega)\left(\int_{\Omega}|\nabla g(x)|^{q} d x\right)^{\frac{1}{q}} .
$$

Combining two previous inequalities we have

$$
\inf _{c \in \mathbb{R}}\left(\int_{\widetilde{\Omega}}|f(y)-c|^{s} d y\right)^{\frac{1}{s}} \leq M_{r, s}(\Omega) B_{r, q}(\Omega)\left\|g \mid L_{q}^{1}(\Omega)\right\| .
$$

By Theorem 2.3

Finally we obtain

$$
\left\|g\left|L_{q}^{1}(\Omega)\left\|\leq K_{p, q}(\Omega)\right\| f\right| L_{p}^{1}(\widetilde{\Omega})\right\| .
$$

$$
\inf _{c \in \mathbb{R}}\left(\int_{\widetilde{\Omega}}|f(y)-c|^{s} d y\right)^{\frac{1}{s}} \leq K_{p, q}(\Omega) M_{r, s}(\Omega) B_{r, q}(\Omega)\left(\int_{\widetilde{\Omega}}|\nabla f|^{p} d y\right)^{\frac{1}{p}} .
$$

It means that

$$
B_{s, p}(\widetilde{\Omega}) \leq K_{p, q}(\Omega) M_{r, s}(\Omega) B_{r, q}(\Omega)
$$

We are ready to establish the main lower estimate:

Theorem 3.4. Let a domain $\Omega \subset \mathbb{R}^{n}$ be a $(r, q)$-Sobolev-Poncaré domain, $1<q<$ $p<r$, and there exists a weak $(p, q)$-quasiconformal homeomorphism $\varphi: \Omega \rightarrow \widetilde{\Omega}$ of a domain $\Omega$ onto a bounded domain $\widetilde{\Omega}$, possesses the Luzin $N$-property and such that

$$
M_{r, p}(\Omega)=\left(\int_{\Omega}|J(x, \varphi)|^{\frac{r}{r-p}} d x\right)^{\frac{r-p}{r p}}<\infty .
$$

Then

$$
\mu_{p}(\widetilde{\Omega}) \geq\left(K_{p, q}^{p}(\Omega) M_{r, p}^{p}(\Omega) B_{r, q}^{p}(\Omega)\right)^{-1} .
$$

This theorem follows from Theorem 3.3 and gives the lower estimate of the first non-trivial Neumann eigenvalue of the $p$-Laplace operator in the terms of $(p, q)$ quasiconformal geometry of domains. 
3.2. The anisotropic Hölder singularities. Define domains $H_{g}$ with anisotropic Hölder $\gamma$-singularities (introduced in [14]):

$$
H_{g}=\left\{x \in \mathbb{R}^{n}: 0<x_{n}<1,0<x_{i}<g_{i}\left(x_{n}\right), i=1,2, \ldots, n-1\right\} .
$$

Here $g_{i}(\tau)=\tau^{\gamma_{i}}, \gamma_{i} \geq 1,0 \leq \tau \leq 1$ are Hölder functions and for the function $G=\prod_{i=1}^{n-1} g_{i}$ denote by

$$
\gamma=\frac{\log G(\tau)}{\log \tau}+1
$$

It is evident that $\gamma \geq n$. In the case $g_{1}=g_{2}=\cdots=g_{n-1}$ we will say that domain $H_{g}$ is a domain with $\sigma$-Hölder singularity, $\sigma=(\gamma-1) /(n-1)$. For $g_{1}(\tau)=g_{2}(\tau)=$ $\cdots=g_{n-1}(\tau)=\tau$ we will use notation $H_{1}$ instead of $H_{g}$.

Define the mapping $\varphi_{a}: H_{1} \rightarrow H_{g}, a>0$, by

$$
\varphi_{a}(x)=\left(\frac{x_{1}}{x_{n}} g_{1}^{a}\left(x_{n}\right), \ldots, \frac{x_{n-1}}{x_{n}} g_{n-1}^{a}\left(x_{n}\right), x_{n}^{a}\right) .
$$

Theorem 3.5. Let $(n-p) /(\gamma-p)<a<p(n-q) / q(\gamma-p)$. Then the mapping $\varphi_{a}: H_{1} \rightarrow H_{g}$, be a weak $(p, q)$-quasiconformal mapping, $1<q<p<\gamma$, from the Lipschitz convex domain $H_{1}$ onto the "cusp"' domain $H_{g}$ with

$$
K_{p, q}\left(H_{1}\right) \leq a^{-\frac{1}{p}} \sqrt{a^{2}\left(\gamma_{1}^{2}+\ldots+\gamma_{n-1}^{2}+1\right)-2 a \sum_{i=1}^{n-1} \gamma_{i}} .
$$

Proof. By simple calculations

$\frac{\partial\left(\varphi_{a}\right)_{i}}{\partial x_{i}}=\frac{g_{i}^{a}\left(x_{n}\right)}{x_{n}}, \quad \frac{\partial\left(\varphi_{a}\right)_{i}}{\partial x_{n}}=\frac{-x_{i} g_{i}^{a}\left(x_{n}\right)}{x_{n}^{2}}+\frac{a x_{i} g_{i}^{a-1}\left(x_{n}\right)}{x_{n}} g_{i}^{\prime}\left(x_{n}\right) \quad$ and $\quad \frac{\partial\left(\varphi_{a}\right)_{n}}{\partial x_{n}}=a x_{n}^{a-1}$

for any $i=1, \ldots, n-1$. Hence $J\left(x, \varphi_{a}\right)=a x_{n}^{a-n} G^{a}\left(x_{n}\right)=a x_{n}^{a \gamma-n}, J\left(x, \varphi_{a}\right) \leq a$ for $a>1$ and

$$
\begin{aligned}
D \varphi_{a}(x)=\left(\begin{array}{ccccc}
x_{n}^{a \gamma_{1}-1} & 0 & \ldots & \left(a \gamma_{1}-1\right) x_{1} x_{n}^{a \gamma_{1}-2} \\
0 & x_{n}^{a \gamma_{2}-1} & \ldots & \left(a \gamma_{2}-1\right) x_{2} x_{n}^{a \gamma_{2}-2} \\
\ldots & \ldots & \ldots & \ldots & \ldots \\
0 & 0 & \ldots & a x_{n}^{a-1}
\end{array}\right) \\
\quad=x_{n}^{a-1}\left(\begin{array}{cccc}
x_{n}^{a \gamma_{1}-a} & 0 & \ldots & \left(a \gamma_{1}-1\right) \frac{x_{1}}{x_{n}} x_{n}^{a\left(\gamma_{1}-1\right)} \\
0 & x_{n}^{a \gamma_{2}-a} & \ldots & \left(a \gamma_{2}-1\right) \frac{x_{2}}{x_{n}} x_{n}^{a\left(\gamma_{2}-1\right)} \\
\ldots & \ldots & \ldots & \ldots \\
0 & 0 & \ldots & a
\end{array}\right) .
\end{aligned}
$$

Because $0<x_{n}<1$ and $x_{1} / x_{n}<1$ we have the following estimate

$$
\begin{aligned}
\left|D \varphi_{a}(x)\right| \leq x_{n}^{a-1} \sqrt{\sum_{i=1}^{n-1}\left(a \gamma_{i}-1\right)^{2}+n-1+a^{2}} & \\
& =x_{n}^{a-1} \sqrt{a^{2}\left(\gamma_{1}^{2}+\ldots+\gamma_{n-1}^{2}+1\right)-2 a \sum_{i=1}^{n-1} \gamma_{i} .}
\end{aligned}
$$


Then

$$
\begin{aligned}
& K_{p, q}\left(H_{1}\right)=\left(\int_{H_{1}}\left(\frac{\left|D \varphi_{a}(x)\right|^{p}}{J\left(x, \varphi_{a}\right)}\right)^{\frac{q}{p-q}} d x\right)^{\frac{p-q}{p q}} \\
& \leq \frac{\sqrt{a^{2}\left(\gamma_{1}^{2}+\ldots+\gamma_{n-1}^{2}+1\right)-2 a \sum_{i=1}^{n-1} \gamma_{i}}}{\sqrt[p]{a}}\left(\int_{H_{1}} x_{n}^{\frac{(p(a-1)-(a \gamma-n)) q}{p-q}} d x\right)^{\frac{p-q}{p q}} \\
& =\frac{\sqrt{a^{2}\left(\gamma_{1}^{2}+\ldots+\gamma_{n-1}^{2}+1\right)-2 a \sum_{i=1}^{n-1} \gamma_{i}}}{\sqrt[p]{a}}\left(\int_{0}^{1} \int_{0}^{x_{n}} \ldots \int_{0}^{x_{n}} x_{n}^{\frac{(p(a-1)-(a \gamma-n)) q}{p-q}} d x_{1} \ldots d x_{n}\right)^{\frac{p-q}{p q}} \\
& =\frac{\sqrt{a^{2}\left(\gamma_{1}^{2}+\ldots+\gamma_{n-1}^{2}+1\right)-2 a \sum_{i=1}^{n-1} \gamma_{i}}}{\sqrt[p]{a}}\left(\int_{0}^{1} x_{n}^{\frac{(p(a-1)-(a \gamma-n)) q}{p-q}+n-1} d x_{n}\right)^{\frac{p-q}{p q}} \\
& \leq \frac{\sqrt{a^{2}\left(\gamma_{1}^{2}+\ldots+\gamma_{n-1}^{2}+1\right)-2 a \sum_{i=1}^{n-1} \gamma_{i}}}{\sqrt[p]{a}},
\end{aligned}
$$

if $(p+a \gamma-p a) q<n p$ or that equivalent $a<p(n-q) / q(\gamma-p)$.

Now we check that $1<q<n p /(p+a \gamma-p a)<p$. The inequality $1<n p /(p+$ $a \gamma-p a)$ implies $a<(n p-p) / \gamma-p$, but

$$
\frac{n p-p}{\gamma-p}<\frac{p(n-q)}{q(\gamma-p)} \text {, if } q>1
$$

The inequality $n p /(p+a \gamma-p a)<p$ implies $a>(n-p) /(\gamma-p)$. So, we have that $a \in((n-p) /(\gamma-p), p(n-q) / q(\gamma-p))$.

We are ready to prove spectral estimates in cusp domains.

Theorem 3.6. Let

$$
H_{g}:=\left\{x \in \mathbb{R}^{n}: n \geq 3,0<x_{n}<1,0<x_{i}<x_{n}^{\gamma_{i}}, i=1,2, \ldots, n-1\right\}
$$

$\gamma_{i} \geq 1, \gamma:=1+\sum_{i=1}^{n-1} \gamma_{i}, g:=\left(\gamma_{1}, \ldots, \gamma_{n-1}\right)$ be domains with anisotropic Hölder $\gamma$-singularities.

Then for $1<p<\gamma$

$$
\frac{1}{\mu_{p}\left(H_{g}\right)} \leq \inf _{a \in I_{a}}\left(a^{2}\left(\gamma_{1}^{2}+\ldots+\gamma_{n-1}^{2}+1\right)-2 a \sum_{i=1}^{n-1} \gamma_{i}\right)^{\frac{p}{2}} B_{r, q}^{p}\left(H_{1}\right),
$$

where $I_{a}=(\max \{(n-p) /(\gamma-p), p(n-q) / \gamma q\}, p(n-q) / q(\gamma-p))$ and $B_{r, q}\left(H_{1}\right)$ is the best constant in the $(r, q)$-Sobolev-Poincaré inequality in the domain $H_{1}$, $q \leq r<\frac{n q}{n-q}$.

Proof. By Theorem 3.5 the mapping $\varphi_{a}: H_{1} \rightarrow H_{g},(n-p) /(\gamma-p)<a<$ $p(n-q) / q(\gamma-p)$,

$$
\varphi_{a}(x)=\left(\frac{x_{1}}{x_{n}} g_{1}^{a}\left(x_{n}\right), \ldots, \frac{x_{n-1}}{x_{n}} g_{n-1}^{a}\left(x_{n}\right), x_{n}^{a}\right) .
$$

maps the convex Lipschitz domain $H_{1}$ onto the cusp domain $H_{g}$ and it is a weak $(p, q)$-quasiconformal mapping, $1<q<p<\gamma$. 
Let us check conditions of Theorem 3.4. Because $\varphi$ is a weak $(p, q)$-quasiconformal mapping then $K_{p, q}\left(H_{1}\right)$ is finite. The basic domain $H_{1}$ is a Lipschitz domains and so is a $(r, q)$-Sobolev-Poincaré domain, i. e. $B_{r, q}\left(H_{1}\right)<\infty$.

Now we estimate the constant $M_{r, p}\left(H_{1}\right)$ :

$$
\begin{aligned}
M_{r, p}\left(H_{1}\right)= & \left(\int_{H_{1}}\left|J\left(x, \varphi_{a}\right)\right|^{\frac{r}{r-p}} d x\right)^{\frac{r-p}{r p}}=a^{\frac{1}{p}}\left(\int_{H_{1}}\left(x_{n}^{a \gamma-n}\right)^{\frac{r}{r-p}} d x\right)^{\frac{r-p}{r p}} \\
= & a^{\frac{1}{p}}\left(\int_{0}^{1}\left(x_{n}^{a \gamma-n}\right)^{\frac{r}{r-p}}\left(\int_{0}^{x_{n}} d x_{1} \ldots \int_{0}^{x_{n}} d x_{n-1}\right) d x_{n}\right)^{\frac{r-p}{r p}} \\
& =a^{\frac{1}{p}}\left(\int_{0}^{1}\left(x_{n}^{a \gamma-n}\right)^{\frac{r}{r-p}} \cdot x_{n}^{n-1} d x_{n}\right)^{\frac{r-p}{r p}}<\infty,
\end{aligned}
$$

if

$$
\frac{(a \gamma-n) r}{r-p}+n-1>-1 \text {, i. e. } a>\frac{n p}{\gamma r} .
$$

Because $0<x_{n}<1$, we have $M_{r, p}\left(H_{1}\right) \leq a^{\frac{1}{p}}$ if $a>n p / \gamma r$. If we take $r<$ $n q /(n-q)$ we obtain that

$$
M_{\frac{n q}{n-q}, p}\left(H_{1}\right) \leq a^{\frac{1}{p}}, \text { if } a>\frac{p(n-q)}{\gamma q} .
$$

The conditions of Theorem 3.4 is fulfilled. Therefore

$$
\begin{aligned}
\frac{1}{\mu_{p}\left(H_{g}\right)} \leq K_{p, q}^{p}\left(H_{1}\right) M_{\frac{n q}{n-q}, p}^{p} & \left(H_{1}\right) B_{\frac{n q}{n-q}, q}^{p}\left(H_{1}\right) \\
& \leq\left(a^{2}\left(\gamma_{1}^{2}+\ldots+\gamma_{n-1}^{2}+1\right)-2 a \sum_{i=1}^{n-1} \gamma_{i}\right)^{\frac{p}{2}} B_{r, q}^{p}\left(H_{1}\right),
\end{aligned}
$$

where $\max \{(n-p) /(\gamma-p), p(n-q) / \gamma q\}<a<p(n-q) / q(\gamma-p)$ and $B_{r, q}\left(H_{1}\right)$ is the best constant in the $(r, q)$-Sobolev-Poincare inequality in the domain $H_{1}$ for some $q \in\left[r, \frac{n q}{n-q}\right)$.

Note that in [23] we proved the estimate of the Poincaré constant in the $(r, q)$ Sobolev-Poincaré inequality in the domain $H_{1}$ :

$$
B_{r, q}\left(H_{1}\right) \leq n\left(\frac{1-\delta}{1 / n-\delta}\right)^{1-\delta} \omega_{n}^{1-\frac{1}{n}}\left(\frac{1}{(n+1) !}\right)^{\frac{1}{n}-\delta}, \delta=\frac{1}{q}-\frac{1}{r} \geq 0 .
$$

The problem of exact values of constants in the $(r, q)$-Sobolev-Poincaré inequalities in the case $p \neq r$ is a complicated open problem even in the case of the unit $\operatorname{disc} \mathbb{D} \subset \mathbb{R}^{2}$.

Acknowledgments. The first author was supported by the United States-Israel Binational Science Foundation (BSF Grant No. 2014055). 


\section{REFERENCES}

[1] K. Astala, P. Koskela, Quasiconformal mappings and global integrability of the derivative, J. Anal. Math. 57 (1991), 203-220.

[2] M. S. Ashbaugh, Isoperimetric and universal inequalities for eigenvalues, Spectral theory and geometry (Edinburgh, 1998), 95-139, London Math. Soc. Lecture Note Ser., 273, Cambridge Univ. Press, Cambridge, 1999.

[3] B. Brandolini, F. Chiacchio, E. B. Dryden, J. J. Langford, Sharp Poincaré inequalities in a class of non-covex sets, arXiv:1608.01236

[4] J. M. Ball, Convexity condition and existence theorems in nonlinear elasticity, Arch. Rational Mech. Anal., 63 (1976), 337-403.

[5] J. M. Ball, Global invertibility of Sobolev functions and the interpenetration of matter, Proc. Roy. Soc. Edinburgh Sect. A, 88 (1981), 315-328.

[6] B. Brandolini, F. Chiacchio, C. Trombetti, Optimal lower bounds for eigenvalues of linear and nonlinear Neumann problems, Proc. of the Royal Soc. of Edinburgh 145A (2015), 31-45.

[7] J. Brennan, The integrability of the derivative in conformal mapping, J. London Math. Soc., 18, (1978), 261-272.

[8] V. I. Burenkov, V. Gol'dshtein, A. Ukhlov, Conformal spectral stability for the Dirichlet-Laplace operator, Math. Nachr., 288 (2015), 1822-1833.

[9] V. I. Burenkov, V. Gol'dshtein, A. Ukhlov, Conformal spectral stability for the Neumann-Laplace operator, Math. Nachr., 289 (2016), 1822-1833.

[10] A. P. Calderón, On an inverse boundary value problem, in Seminar on Numerical Analysis and its Applications to Continuum Physics (Rio de Janeiro, 1980), 65-73.

[11] M. Csörnyei, S. Hencl, J. Malý, Homeomorphisms in the Sobolev space $W^{1, n-1}$, J. Reine Angew. Math. 644 (2010), 221-235.

[12] V. Ferone, C. Nitsch and C. Trombetti, A remark on optimal weighted Poincaré inequalities for convex domains, Atti Accad. Naz. Lincei Rend. Lincei Mat. Appl., 23 (2012), 467-475.

[13] F. W. Gehring, The $L_{p}$-integrability of the partial derivatives of a quasiconformal mapping, Acta Math., 130 (1973), 265-277.

[14] V. Gol'dshtein, L. Gurov, Applications of change of variables operators for exact embedding theorems, Integral Equations Operator Theory 19 (1994), 1-24.

[15] V. Gol'dshtein, L. Gurov, A. Romanov, Homeomorphisms that induce monomorphisms of Sobolev spaces, Israel J. Math., 91 (1995), 31-60.

[16] V. Gol'dshtein, V. Pchelintsev, A. Ukhlov, Spectral Estimates of the p-Laplace Neumann operator and Brennan's Conjecture, Boll. Unione Mat. Ital. (2017).

[17] V. M. Gol'dshtein, Yu. G. Reshetnyak, Quasiconformal mappings and Sobolev spaces, Dordrecht, Boston, London: Kluwer Academic Publishers, 1990.

[18] V. M. Gol'dshtein, A. S. Romanov, Mappings preserving Sobolev spaces, Siberian Math. J., 25 (1984), 55-61.

[19] V. Gol'dshtein, A. Ukhlov, Weighted Sobolev spaces and embedding theorems, Trans. Amer. Math. Soc., 361, (2009), 3829-3850.

[20] V. Gol'dshtein, A. Ukhlov, About homeomorphisms that induce composition operators on Sobolev spaces, Complex Var. Elliptic Equ. 55 (2010), 833-845.

[21] V. Gol'dshtein, A. Ukhlov, On the first Eigenvalues of Free Vibrating Membranes in Conformal Regular Domains. Arch. Rational Mech. Anal., 221 (2016), no. 2, 893-915.

[22] V. Gol'dshtein, A. Ukhlov, Spectral estimates of the $p$-Laplace Neumann operator in conformal regular domains. Transactions of A. Razmadze Math. Inst., 170(1) (2016), $137-148$.

[23] V. Gol'dshtein, A. Ukhlov, The spectral estimates for the Neumann-Laplace operator in space domains. Adv. in Math., 315 (2017), 166-193.

[24] C. Enache, G. A. Philippin, On some isoperimetric inequalities involving eigenvalues of symmetric free membranes, ZAMM Z. Angew. Math. Mech., 95 (2015), 424-430.

[25] P. Hajlasz, P. Koskela, Isoperimetric inequalities and imbedding theorems in irregular domains, J. London Math. Soc., 58 (1998), 425-450.

[26] J. Heinonen, T. Kilpelinen, O. Martio, Nonlinear Potential Theory of Degenerate Elliptic Equations. Clarendon Press. Oxford, New York, Tokio. 1993. 
[27] S. Hencl, P. Koskela, Composition of quasiconformal mappings and functions in Triebel-Lizorkin spaces, Math. Nachr. 286 (2013), 669-678.

[28] L. Esposito, C. Nitsch, C. Trombetti, Best constants in Poincaré inequalities for convex domains, J. Convex Anal., 20, 253-264 (2013).

[29] L. Kleprlik, Mappings of finite signed distortion: Sobolev spaces and composition of mappings. J. Math. Anal. Appl., 386 (2012), 870-881.

[30] P. Koskela, J. Onninen, J. Tyson, Quasihyperbolic boundary conditions and capacity: Hölder continuity of quasiconformal mappings, Comment. Math. Helv. 76 (2001), 416-435.

[31] P. Koskela, J. Onninen, J. Tyson, Quasihyperbolic boundary conditions and PoincarÃC) domains, Math. Ann. 323 (2002), 811-830.

[32] H. Koch, P. Koskela, E. Saksman,T. Soto, Bounded compositions on scaling invariant Besov spaces, J. Funct. Anal. 266 (2014), 2765-2788.

[33] P. Koskela, D. Yang, Y. Zhou, Pointwise characterizations of Besov and TriebelLizorkin spaces and quasiconformal mappings. Adv. Math. 226 (2011), 3579-3621.

[34] P. Koskela, J. Xiao, Yi Ru-Ya Zhang, Y. Zhou, A quasiconformal composition problem for the $Q$-spaces, J. Eur. Math. Soc. (JEMS) 19 (2017), 1159-1187.

[35] V. I. Kruglikov, Capacities of condensors and quasiconformal in the mean mappings in space. Mat. Sb. (N.S.) 130(172) (1986), 185-206.

[36] R. S. Laugesen, C. Morpurgo, Extremals of Eigenvalues of Laplacians under Conformal Mapping, Journal Func. Analysis, 155 (1998), 64-108.

[37] I. G. Markina, The change of variable that preserves the differential properties of functions, Siberian Math. J., 31 (1990), 73-84.

[38] O. Martio, V. Ryazanov, U. Srebro, E. Yakubov, Moduli in modern mapping theory. Springer Monographs in Mathematics. Springer, New York, 2009.

[39] O. Martio, J. Väisälä, Global $L_{p}$-integrability of the derivative of a quasiconformal mapping, Complex Variables Theory Appl. 9 (1988), 309-319.

[40] V. G. Maz'ya, Weak solutions of the Dirichlet and Neumann problems, Trudy Moskov. Mat. Ob-va., 20 (1969), 137-172 (1969)

[41] V. Maz'ya, Sobolev spaces: with applications to elliptic partial differential equations, Springer, Berlin/Heidelberg, 2010.

[42] V. Maz'ya, T. O. Shaposhbikovs, Multipliers in Spaces of Differentiable Functions, Leningrad Univ. Press., 1986.

[43] M. Oliva, M. Prats, Sharp bounds for composition with quasiconformal mappings in Sobolev spaces, J. Math. Anal. Appl. 451 (2017), 1026-1044.

[44] L. E. Payne, H. F. Weinberger, An optimal Poincaré inequality for convex domains, Arch. Rat. Mech. Anal., 5 (1960), 286-292.

[45] R. R. Salimov, Lower Q-homeomorphisms with respect to the p-modulus, Ukr. Mat. Visn. 12 (2015),484-510.

[46] E. Sevost'yanov, On open and discrete mappings with a modulus condition, Ann. Acad. Sci. Fenn. Math. 41 (2016), 41-50.

[47] G. Szegö, Inequalities for certain eigenvalues of a membrane of given area, J. Rational Mech. Anal., 3 (1954), 343-356.

[48] A. Ukhlov, On mappings, which induce embeddings of Sobolev spaces, Siberian Math. J., 34 (1993), 185-192.

[49] A. Ukhlov, S. K. Vodop'yanov, Mappings with bounded ( $P, Q)$-distortion on Carnot groups, Bull. Sci. Math. 134 (2010), 605-634.

[50] S. K. Vodop'yanov, Taylor Formula and Function Spaces, Novosibirsk Univ. Press., 1988.

[51] S. K. Vodop'yanov, Mappings of homogeneous groups and embeddings of function spaces, Siberian Math. J., 30 (1989), 25-41.

[52] S. K. Vodop'yanov, $L_{p}$-theory of potential and quasiconformal mappings on homogeneous groups, Sovrem. Probl. Geom. Analiz., 263 (1990), 45-89.

[53] S. K. Vodop'yanov, Topological and geometric properties of mappings with an integrable Jacobian in Sobolev classes, Siberian Math. J., 41 (2000), 19-39.

[54] S. K. Vodop'yanov, V. M. Gol'dshtein, Structure isomorphisms of spaces $W_{n}^{1}$ and quasiconformal mappings, Siberian Math. J., 16 (1975), 224-246. 
[55] S. K. Vodop'yanov, V. M. Gol'dshtein, Functional characteristics of quasi-isometrical mappings, Siberian Math. J. 17, (1976), 758-773.

[56] S. K. Vodop'yanov, V. M. Gol'dshtein, Yu. G. Reshetnyak, On geometric properties of functions with generalized first derivatives, Uspekhi Mat. Nauk 34 (1979), 17-65.

[57] Vodop'yanov S. K., Ukhlov A. D. Sobolev spaces and $(P, Q)$-quasiconformal mappings of Carnot groups, Siberian Math. J. 39 (1998), 665-682.

[58] S. K. Vodop'yanov, A. D. Ukhlov, Superposition operators in Sobolev spaces, Russian Mathematics (Izvestiya VUZ) 46 (2002), no. 4, 11-33.

[59] H. F. Weinberger, An isoperimetric inequality for the $n$-dimensional free membrane problem, Arch. Rat. Mech. Anal., 5 (1956), 633-636. 